\title{
Electrocoagulation with AC Electrical Current at Low Voltage for Separation of Crude Glycerol from Biodiesel Product Mixture
}

\author{
Warakorn Sakkamas ${ }^{1}$, Ajalaya Boripun ${ }^{1}$, Rossarin Ampairojanawong ${ }^{1}$, Sayan Ruankon ${ }^{2}$, Thanapong Suwanasri ${ }^{2}$, and \\ Tawiwan Kangsadan ${ }^{1, *}$ \\ ${ }^{1}$ Chemical and Process Engineering Program, The Sirindhorn International Thai-German Graduate School of Engineering (TGGS), \\ King Mongkut's University of Technology North Bangkok, Bangkok, Thailand \\ ${ }^{2}$ Electrical Power and Energy Engineering Program, The Sirindhorn International Thai-German Graduate School of Engineering \\ (TGGS), King Mongkut's University of Technology North Bangkok, Bangkok, Thailand
}

\begin{abstract}
Electrocoagulation with AC electrical current at low voltage was implemented to remove crude glycerol from biodiesel which was produced via transesterification reaction of refined palm oil (RPO) as feedstock with methanol in the presence of sodium hydroxide derivative-catalyst at $60^{\circ} \mathrm{C}$ for $2 \mathrm{hr}$ using the conventional heating in the water bath. Effects of point-to-point electrode configuration, electrode materials, inter-electrode distances, optimized AC low voltages, molar ratios of glycerol and biodiesel product mixture on the separation time and the separation efficiency were studied. Electrocoagulation process with applied $\mathrm{AC}$ at $96 \mathrm{~V}$ and using $\mathrm{Al}$ point-to-point electrodes at the inter-electrode distance of $0.1 \mathrm{~cm}$ could efficiently remove free glycerol more than the gravitation settling for the separation time of $120 \mathrm{~s}$. The separation efficiency was over $99.99 \%$. Even though the clear interface between biodiesel and glycerol was firstly observed after applying the electrocoagulation for $30 \mathrm{~s}$, the separation time had to proceed for additional $90 \mathrm{~s}$ to eliminate unreacted catalyst. The methyl ester content of $98.56 \pm 0.47 \mathrm{wt} \%$ was obtained after purification with 2 times of water-washing. This process can be achieved by shortening the separation time and could significantly reduce the water consumption during the purification process.
\end{abstract}

\section{Introduction}

Global energy consumption is getting higher annually for the country development and the industrial production. Typically, fossil fuel has been used in petrochemical production to produce fuel, electricity, chemical feedstock etc. Oil fuel is the main product from petrochemical production, and it is essentially consumed for transportation sector over $80 \%$ [1], however, fossil fuel is non-renewable energy and it generates a ton of pollutants such as carbon monoxide, carbon dioxide, sulfur, nitrogen dioxide and various harmful gases. Moreover, those pollutants can cause global warming and acid rain that are serious problem in nowadays. Hence, renewable fuel is more capable for long-term used. Biodiesel is one of interesting renewable fuel. It can be produced from palm, seaweed, coconut and other oil plants. It can achieve economic sustainability and environment-friendly [2].

Commercially, high yield of biodiesel production is essential to achieve high profit. Thus, not only reaction process is supposed to be at high efficiency, but separation section also needs to be carried out. In conventional purification process, many methods have developed such as wet washing, dry washing, centrifuge and gravitation. Those techniques are proven in various studies but there are some limitations to be solved for example, product loss, high investment cost, long separation time and plenty waste.

In recent year, many researches have been developed to accomplish more efficient and environment-friendly separation process. Ceramic membrane was implemented to purify glycerol and soap from biodiesel [3]. The pore size of membrane was $0.05 \mu \mathrm{m}$. Crude biodiesel without methanol was injected through the membrane. Soap and glycerol were collecting and agglomerating to larger particles. Therefore, only biodiesel could permeate through the membrane pore. After treating biodiesel, this process can produce $97.85 \%$ purity of biodiesel.

Electrostatic separation of glycerol and waste cooking oil was studied by applying direct current at low voltage [4]. This research demonstrated that $33.5 \mathrm{~V}$ was applied through stainless steel electrodes in a 3-L tank. Glycerol was separated by $10 \%$ of total volume within $130 \mathrm{~s}$ and it was observed that high voltage had more purification efficiency.

Effect of direct (DC) and alternative current (AC) applied in waste water purification with bio-charcoal were investigated [5]. The results shown that removal efficiency using both current types were insignificantly different. Nevertheless, the purification process with AC could save more energy consumption about 2 times. After operating with DC for a long period of time,

\footnotetext{
Corresponding author: tawiwan.k@tggs.kmutnb.ac.th
} 
electrodes were consumed and caused oxide film on electrodes. This could reduce the removal efficiency.

Most of researches used plate or bar electrodes and studied on particular one volume ratio $[4,6,7]$. In order to improve efficiency and analyze capability of electrocoagulation (EC), this research studied the point to point electrode arrangement varying the volume ratio of biodiesel to oil [9]. In fact, not only high efficiency is supposed to be concerned, large production scale is also important. Thus, the objective of this research is to investigate the effect of EC with applied $\mathrm{AC}$ electrical current at low voltage for the separation of biodiesel and crude glycerol in order to purify biodiesel final product. Correlation of voltages, electrode materials, inter-electrode distances, and volume ratios of biodiesel and glycerol were determined. The purified biodiesel was analyzed by titration and gas chromatography in order to realize the remaining soap content and the methyl ester content, respectively. Finally, the performance of electrocoagulation technology was compared to conventional separation by gravitation settling (GS).

\section{Materials and methods}

\subsection{Materials}

Fatty acids composition of refined palm oil (RPO) used as a raw material are shown in Table 1 . The chemical reagents are methanol with $99.9 \%$ purity, sodium hydroxide derivative-catalyst and $\mathrm{n}$-heptane manufactured by $\mathrm{RCI}$ Labscan Limited. All standard chemicals used for the biodiesel analysis according to EN $14103[8,9]$ and EN 14105 [10] were supplied by the Sigma-Aldrich Chemicals Company.

Table 1. Fatty acids composition of refined palm oil (RPO) obtained from gas chromatography analysis.

\begin{tabular}{|c|c|}
\hline Fatty acid & Composition $(\% \mathbf{\%} / \mathbf{w})$ \\
\hline $\mathrm{C} 12: 0$ & 0.80 \\
\hline $\mathrm{C} 14: 0$ & 1.10 \\
\hline $\mathrm{C} 16: 0$ & 43.45 \\
\hline $\mathrm{C} 18: 0$ & 4.23 \\
\hline $\mathrm{C} 18: 1$ & 40.90 \\
\hline $\mathrm{C} 18: 2$ & 9.10 \\
\hline $\mathrm{C} 18: 3$ & 0.24 \\
\hline
\end{tabular}

\subsection{Methods}

The experimental procedures were divided into 3 parts: (1) the production of biodiesel and its by-product glycerol; (2) the separation of glycerol from biodiesel; and (3) the analysis of final products.

\subsubsection{Transesterification reaction process}

Biodiesel and by-product glycerol were produced via homogeneous catalyzed transesterification reaction using conventional heating in the water bath as shown in
Fig. 1. The reaction was carried out in 2 flask-neck glass reactors placed in the water bath which was used to heat samples under control temperature. The reactor was equipped with the mechanical overhead stirrer and the condenser to reduce the losses of methanol due to evaporation during conversion process. An initial mass of $500 \mathrm{~g}$ of refined palm oil (RPO) was preheated in a thermal bath until the temperature reached to $60^{\circ} \mathrm{C}$ before adding the methanol and catalyst solution. The reaction operated at the optimum condition which obtained from previous study [11-13] which the molar ratio of methanol to oil of $6: 1$ with the $\mathrm{NaOH}$-derivative catalyst concentration of $2 \% \mathrm{w} / \mathrm{v}$ while the stirrer speed was fixed at $480 \mathrm{rpm}$. The reaction was carried out at $60^{\circ} \mathrm{C}$ using conventional heating and at atmospheric pressure. To ensure the complete reaction, the reaction time was proceeded for $2 \mathrm{hr}$. At the end of reaction, the product mixture contains biodiesel, glycerol, methanol, remaining catalyst and unreacted reactants. The product mixture was first separated by using the gravitational settling which the product mixture was poured into a separating funnel and let it settle until the clear interface between biodiesel and glycerol layers were clearly observed. This would ensure that glycerol was completely separated from the biodiesel. These two products were collected separately. The required total volume of approximately $400 \mathrm{~mL}$ would be used for the separation experiments using the GS and the EC techniques.

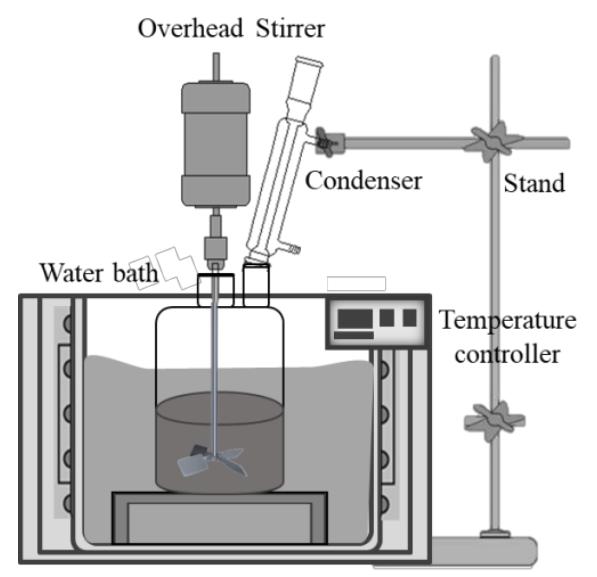

Fig. 1. Schematic diagram of biodiesel production via homogeneous catalyzed transesterification reaction using conventional heating in the water bath.

\subsubsection{Gravitational settling separation process}

The biodiesel and glycerol product samples collected in the previous step were heated at $60^{\circ} \mathrm{C}$ separately until the sample were homogeneous and then immediately poured these two samples in the cylindrical chamber (diameter of $8.0 \mathrm{~cm}$ and height of $15 \mathrm{~cm}$ ) as shown in Fig. 2 (a). The volume of biodiesel and glycerol were according the volume ratio of biodiesel and glycerol with the required total volume of approximately $400 \mathrm{~mL}$. The volume ratios of biodiesel and glycerol at 7:1, 6:2, 5:3, $4: 4,3: 5$, and 2:6 were investigated. The product mixture was separated by gravitational settling until 
the clear interface between biodiesel and glycerol layers was observed. The separation efficiency was determined, and the purity of biodiesel and remaining soap content were analyzed.

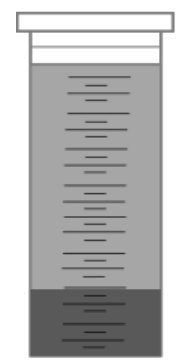

(a)

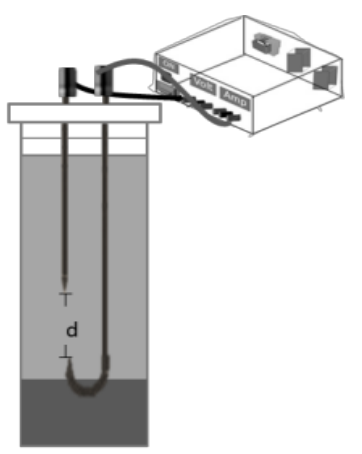

(b)
Fig. 2. Schematic diagram of glycerol separation from biodiesel using (a) the gravitational settling (GS) and (b) the electrocoagulation process (EC).

\subsubsection{Electrocoagulation separation process}

In principle, a glycerol droplet consists of intermolecular and surface forces, van der Waals (vdW) and electric double layer in balance. EC process can promote colloid destabilization mechanism in which the electric field between electrodes has potential to generate electron and decrease electric double layer of droplet which is repulsive force. So, van der Waals force was dominating at high ion concentration and able to attract droplets being larger size. After that glycerol molecules had moved closer and linked together. Finally, they had precipitated to the bottom [14-16]. Additionally, AC was applied to reduce corrosion of electrodes that can impact to efficiency loss of current transfer [17].

The product mixture of biodiesel and glycerol was prepared with the same procedure as mentioned in the previous section. The same set of volume ratios of biodiesel and glycerol was investigated. However, the product mixture was separated by electrocoagulation as shown in Fig. 2(b) and 3. The separation was completed when the clear interface between biodiesel and glycerol layers was observed. The separation efficiency was determined, and the purity of biodiesel and remaining soap content were analyzed.

Experiments were performed in the electrocoagulation cylinder setup and the volume of the solution of each run for EC separation was approximately $400 \mathrm{~mL}$. The unit consist of one pair of electrodes were connected to the power source and the diameter of each electrode was $3.2 \mathrm{~mm}$. Low voltage (up to $100 \mathrm{~V}$ ) EC using point-to-point electrodes-configuration which demonstrated the highest efficiency (data not shown). AC power supply are having an input of $220 \mathrm{~V}$ and step up with two transformer with variable output of $24 \mathrm{~V}, 48 \mathrm{~V}, 72 \mathrm{~V}$ and $96 \mathrm{~V}$ with low current of ampere was used as direct current source with run reaction of EC separation at room temperature. As presented in Fig. 3, the instrument setup mainly in control box consisted of two step-up transformers, AC volt meter, AC amp meter, cable connector and resistor.

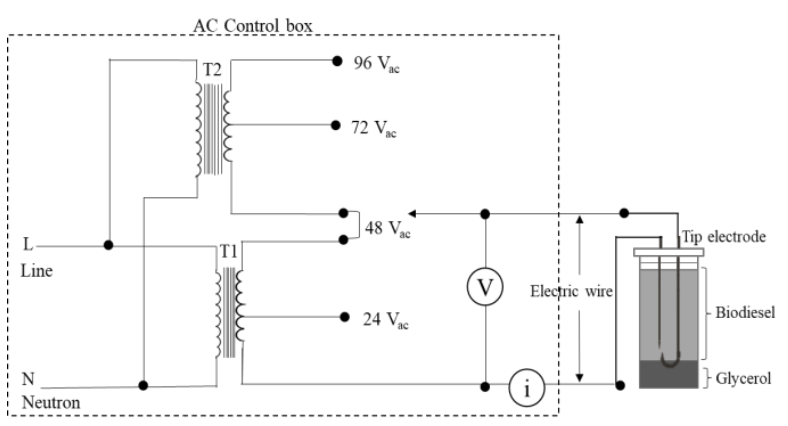

Fig. 3. Schematic diagram of glycerol separation from biodiesel using EC with $\mathrm{AC}$ electrical current at low voltage.

\subsection{Analysis methods}

The separated biodiesel and glycerol product samples were analyzed for the separation efficiency of individual separation technique based on the separation of glycerol and then later comparing the performance. Furthermore, the biodiesel product was analyzed for its purity as methyl ester content (\%) using Gas Chromatography (GC) according to the standards EN $14103[8,9]$ and EN $14105[10]$ as well as remaining soap content (ppm).

\subsubsection{Separation efficiency}

For measuring removal glycerol from biodiesel to improve efficient performance of separation technique so the volumetric percentage separation calculations $(\% \mathrm{v} / \mathrm{v})$ were performed [18]. However, the separation efficiency calculation can also be performed on a mass basis $(\% \mathrm{w} / \mathrm{w})$. The percentage of $\eta_{g}$ calculation was expressed as followed equation (1).

$$
\eta_{g}(\%)=\left(\frac{V_{f}}{V_{i}}\right) \times 100 \%
$$

where $V_{i}$ is initial volume of glycerol and $V_{f}$ is final volume of glycerol after the separation.

\subsubsection{Purity of biodiesel}

Once separation was carried out. All samples were taken by pipetting from the top phase which consisted of biodiesel. In order to test purity of biodiesel according to the standard EN14103 [8] by using SHIMADZU GC2010 plus equipped with FID detector and DB-WAX column with a dimension of $30 \mathrm{~m} \times 0.25 \mathrm{~mm}$ I.D. coated with $0.25 \mu \mathrm{m}$ film and FID detector was set at $250^{\circ} \mathrm{C}$ with nitrogen as carrier gas. Moreover, the concentration of free glycerol, mono-, di-, triglyceride, methanol and biodiesel were analyzed by using a SHIMADZU GC-2010 Plus series high-end gas chromatograph system which is equipped with FID detector and HT-5 column with a dimension of $25 \mathrm{~m} \times 0.22 \mathrm{~mm}$ I.D. coated with $0.1 \mu \mathrm{m}$ film according to the standard EN 14105 [12]. 


\subsubsection{Remaining soap content}

The remaining soap content was analyzed by pipetting $40 \mathrm{~g}$ of biodiesel from the top phase after each desired experiment on the separation time were completed. To ensure the accuracy of remaining soap contents at each separation time, the biodiesel-glycerol mixtures were prepared individually for each separation time experiment. So that the ratio of biodiesel and glycerol mixture were the same. Then, the extracted sample was blended with $98 \%$ acetone in water. Then biodiesel mixture was titrated with $0.1 \mathrm{~N} \mathrm{HCl}$ along with $0.4 \%$ Bromophenol Blue in water as indicator [19-21]. Titration was performed until equilibrium point. The mixture color was going to be changed from blue to yellow. Finally used $\mathrm{HCl}$ was recorded for remaining soap calculation as shown in equation (2).

$$
m_{\text {soap }}=\frac{V_{H c l} \times 0.01 \times M W_{\text {catalyst }} \times 1000}{m_{\text {sample }}}
$$

where $m_{\text {soap }}$ is the amount of soap remaining content (ppm), $m_{\text {sample }}$ is the weight of biodiesel sample (g), $V_{H c l}$ is the volume of added hydrochloric acid until solution change color from yellow to blue-violet $(\mathrm{mL})$ and $M W_{\text {soap }}$ is the molecular weight of soap.

\section{Results and discussions}

The separation methods using GS and EC were studied for the separation of glycerol from biodiesel. The effect of volume ratio of biodiesel and glycerol on the separation time as well as the separation efficiency was investigated. The purity of biodiesel and remaining soap content were determined and compared.

\subsection{Gravitational settling separation process}

The separation of glycerol from biodiesel using GS was studied varying volume ratios of biodiesel and glycerol. The separation time as well as the separation efficiency were determined to compare with EC separation process.

\subsubsection{Effect of volume ratio}

Since the total volume of EC cylindrical chamber was $401.92 \mathrm{~mL}$, by dividing into 8 portions, the volume ratios of biodiesel to glycerol were assigned for $7: 1,6: 2$, $5: 3,4: 4,3: 5$ and $2: 6$. For the volume ratio of $7: 1$, the crude biodiesel of $351.68 \mathrm{~mL}$ and the crude glycerol of $50.24 \mathrm{~mL}$ were added and mixed into the EC cylindrical chamber. Fig. 4 showed the effect of volume ratios of biodiesel to glycerol. The separation efficiency was increased as the separation time increased because initially there was a large amount of glycerol and more possibility to coagulate into a bigger size of glycerol. After that smaller suspending particles such as monoglyceride and diglyceride which were not converted slightly precipitated. Finally, the separation efficiency reached $99.99 \%$ at all volume ratios. However, the volume ratio of biodiesel to glycerol was inversely proportional to the separation time and the separation efficiency. The fastest time of separation condition was at the volume ratio of 7:1 (low amount of crude glycerol). Glycerol was completely separated at $200 \mathrm{~s}$. The highest time of separation condition was the ratio of $3: 5$ and reached $99.99 \%$ separation efficiency at 400 s. One step of increasing glycerol ratio effected to completion of separation time by $40-60 \mathrm{~s}$ for the reason that high glycerol volume influenced the viscosity of solution to become more viscous. So, particles moved lower than in a low-viscosity solution by GS.

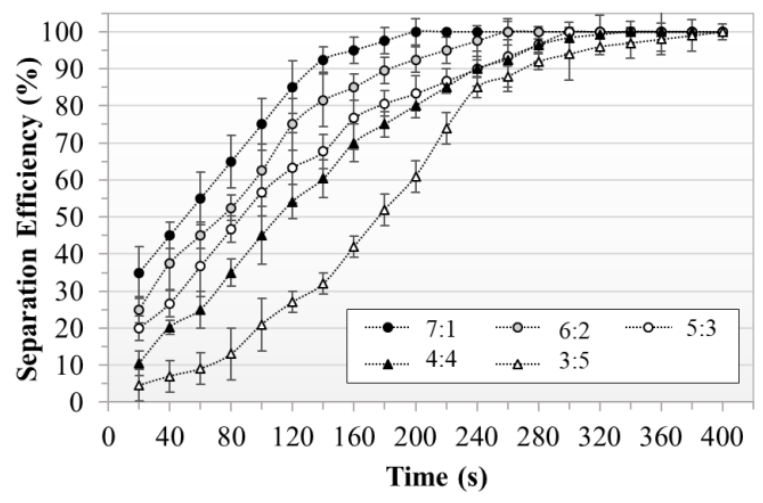

Fig. 4. Separation efficiency and separation time with the use of GS varying volume ratios of biodiesel to glycerol.

\subsection{Electrocoagulation process}

Alternative separation technique using EC was investigated to separate the glycerol from biodiesel. The optimum conditions for EC process were determined for the type of electrode material, the electrodes arrangement and the distance between inter-electrodes were studied. Moreover, the effect of volume ratio on the separation time and its efficiency was determined. The purity of biodiesel and the remaining soap content were analyzed.

\subsubsection{Effect of volume ratio}

EC was applied to shorten the complete sedimentation time or the separation time. Fig 5 shows that EC could reduce the separation time by 4-6 times depending on the volume ratio of biodiesel to glycerol. The separation efficiency profile with the use of EC was in similar trend as in GS. The fastest precipitation occurred at the 7:1 volume ratio of biodiesel to glycerol with crude glycerol absolutely separated at $30 \mathrm{~s}$. Since EC separation process depends on the conductivity of particles, in this study are glycerol, catalyst and methanol by destabilization. The generated electrical field induces the ionic strength, charge neutralization, inter-droplet cross-link of these molecules [21]. Then, these particles are precipitated to the bottom. In this study, the separation efficiency at the volume ratio of biodiesel to glycerol 5:3 had been higher than $6: 2$ until $20 \mathrm{~s}$ because large amount of glycerol had moved to the electric field generated between electrodes and then coagulated [22]. In fact, not only electrocoagulation takes place in the system, but the gravity also forces molecules to settle to the bottom. Therefore, most of 
molecules had been settled with the influence of EC in the first $20 \mathrm{~s}$. After that the gravitational force had interfered the performance of EC. Finally, the gravitational force took over the sedimentation phenomena. On the other hand, volume ratios of 4:4 and 3:5 behaved differently due to the viscosity of solution. The molecules were not freely moved to receive charges.

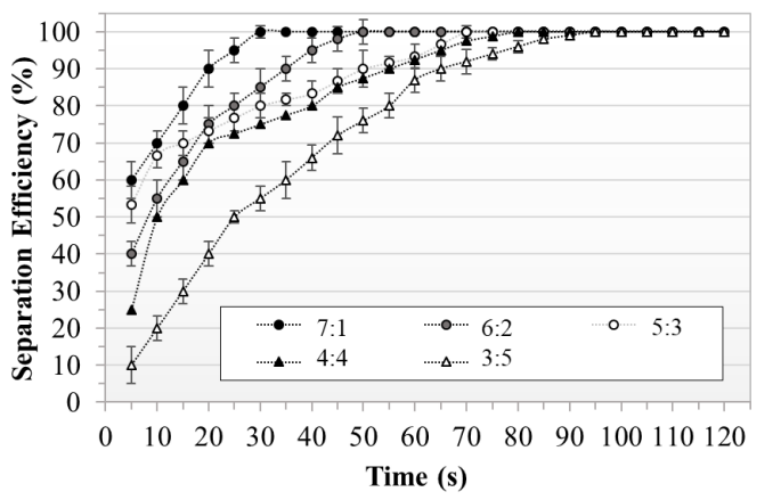

Fig. 5. Separation efficiency and separation time with the use of EC with applied $\mathrm{AC}$ voltage of $96 \mathrm{~V}$ using $\mathrm{Al}$ point-to-point electrodes and the inter-electrode distance of $0.1 \mathrm{~cm}$ varying volume ratios of biodiesel to glycerol.

\subsubsection{Effect of applied voltage}

Typically for $100 \mathrm{~g}$ of biodiesel, approximately $10 \mathrm{~g}$ of crude glycerol are produced which is equivalent to the volume ratio of biodiesel to glycerol 5:0.5. At this low volume of glycerol, the phenomena could not be clearly observed during EC with applied AC electrical current at low voltage. Therefore, higher volume ratio of biodiesel to glycerol 5:3 was required to observe the effect of volume of ratio under the influence of low voltage in the range of 48, 72 and $96 \mathrm{~V}$ as shown in Fig. 6. The phenomena of glycerol particles movement to the tip electrode was clearly observed only at $96 \mathrm{~V}$. In order to observe the phenomena, more glycerol content was required.

EC with applied $\mathrm{AC}$ at $96 \mathrm{~V}$ was more competitive than the one at $72 \mathrm{~V}$ and $48 \mathrm{~V}$ for the first $30 \mathrm{~s}$. After that the performance at $96 \mathrm{~V}$ and $72 \mathrm{~V}$ were not significantly different. However, EC with applied AC at $48 \mathrm{~V}$ took $110 \mathrm{~s}$ to complete the separation because the electric stress between electrodes was not sufficient to pull molecules together. Lower applied AC voltage at 24 $\mathrm{V}$ was conducted but the performance was relatively the same as the one with GS.

\subsubsection{Effect of distance between electrode tips}

Electrodes configuration of point-to-point was used in this experiment varying the inter-electrode distance $(0.1$, $0.2,0.4,0.6$ and $0.8 \mathrm{~cm})$. The electrical field was generated in this region between electrodes. Effects of inter-electrode distance on the separation efficiency and the separation time were shown in Fig. 7. The shortest separation time was $70 \mathrm{~s}$ for the inter-electrode distance of $0.1 \mathrm{~cm}$. The separation efficiency was decreased as the inter-electrode distance increased. Small gap between electrode tips created more electric field stress [23]. Generally, electrons move through medium which is mainly biodiesel and glycerol. Biodiesel is non-polar and non-conductive while glycerol is conductive. Therefore, electrons need to move through glycerol to another electrode. If the gap is small, electric field stress is high because electrons are easily moved. Then, glycerol and other conductive particles moved to the electric field and became flocculation.

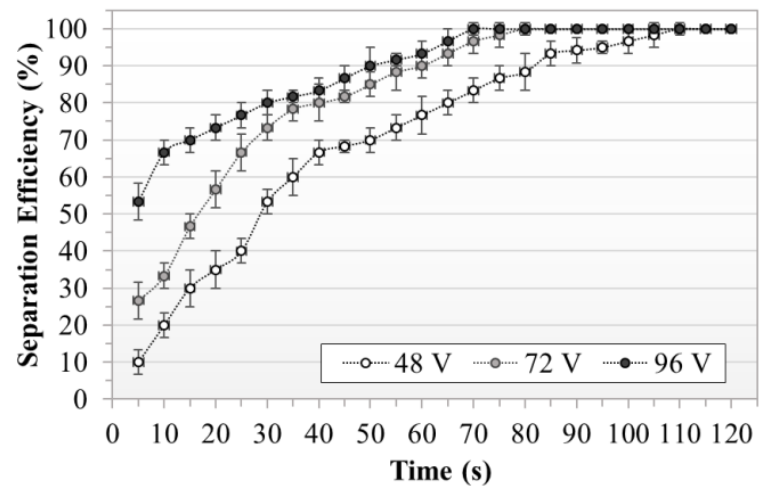

Fig. 6. Separation efficiency and separation time with the use of EC using Al point-to-point electrodes and the inter-electrode distance of $0.1 \mathrm{~cm}$ for volume ratio of biodiesel to glycerol of 5:3 varying applied $\mathrm{AC}$ voltages.

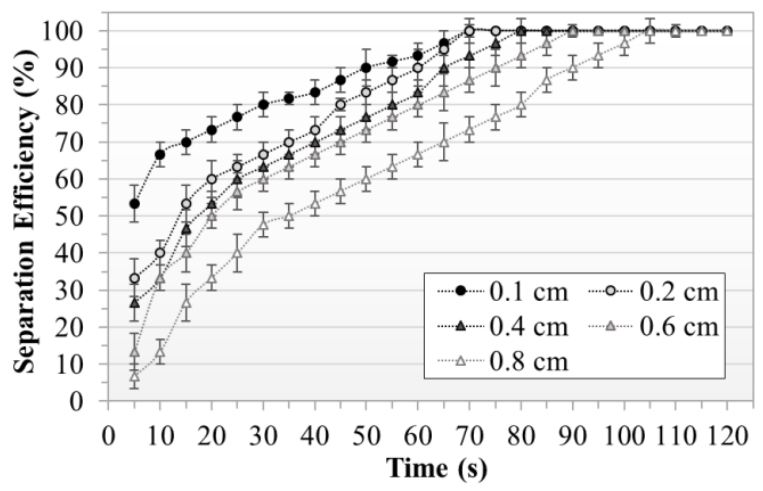

Fig. 7. Separation efficiency and separation time with the use of EC with applied AC voltage of $96 \mathrm{~V}$ using Al point-to-point electrodes for volume ratio of biodiesel to glycerol of 5:3 varying inter-electrode distances.

\subsubsection{Effect of electrode material}

The suitable electrode material for EC should have high conductivity and non-corrosion. The selected materials for this study were iron ( $\mathrm{Fe})$ and aluminum (Al). The separation performance of these two electrode materials were presented in Fig. 8. Al-Al electrodes performed better during the initial separation time of $40 \mathrm{~s}$ because $\mathrm{Al}$ has 3 times higher electro conductivity $\left(3.5 \times 10^{7}\right)$ than $\mathrm{Fe}$. Another reason is that $\mathrm{Al}$ can produce more reaction pathways with the production of several species of aluminum hydroxide [24]. After $40 \mathrm{~s}$, the separation efficiencies of both electrode materials were not significantly different and relatively completed the separation at the same time. Practically, both materials can be used for the electrocoagulation, but $\mathrm{Al}$ is more 
suitable in terms of less corrosive. Then, the life time of electrode can be extended, and the investment cost can be reduced.

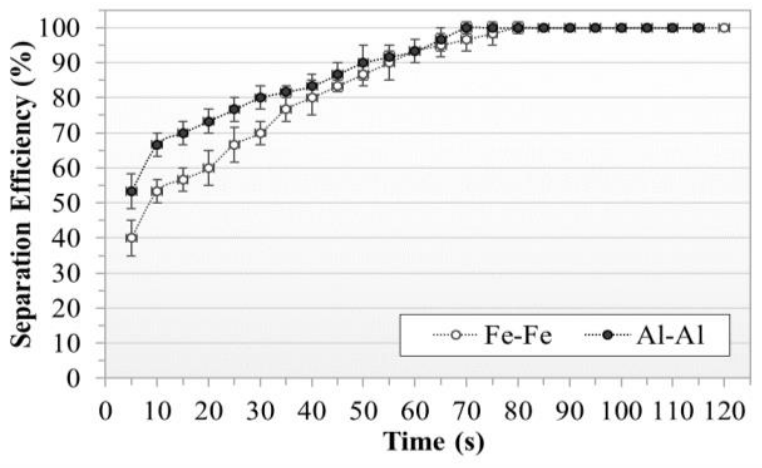

Fig. 8. Separation efficiency and separation time with the use of EC with applied AC voltage of $96 \mathrm{~V}$ using point-to-point $\mathrm{Fe}-\mathrm{Fe}$ and $\mathrm{Al}-\mathrm{Al}$ electrodes and the inter-electrode distance of $0.1 \mathrm{~cm}$ for volume ratio of biodiesel to glycerol of $5: 3$.

\subsubsection{Effect of methyl ester content}

Apparently, EC with applied AC low voltage at $96 \mathrm{~V}$ was the best condition to achieve the methyl ester content of $96 \%$ within the separation time of $70 \mathrm{~s}$ as shown in Fig. 9.



Fig. 9. Separation efficiency and the methyl ester content before the purification process of water-washing with the use of EC using Al point-to-point electrodes and the inter-electrode distance of $0.1 \mathrm{~cm}$ for volume ratio of biodiesel to glycerol of $5: 3$ varying applied $\mathrm{AC}$ voltages.

By decreasing voltage, methyl ester content was lower, and the separation time was increased. Glycerol and other contaminants would receive charges between electrode points afterwards they were linked to large molecules and settled to the bottom [21]. Therefore, high voltage can excite electron movement around the electric field and several aluminum hydroxide species were produced [25].

\subsubsection{Effect of remaining soap content}

Even biodiesel is suitable for diesel engine with low harmful exhaust gas but contaminating soap in biodiesel can cause various problems such as cumulating and clogging up in fuel filter which this causes engine malfunction and it can shake while driving. If it was used under heavy load, this may be stalled. Thus, remaining soap content is supposed to be less than $5 \mathrm{ppm}$ after washing step following ASTM D6751. With EC separation technique, the remaining soap in biodiesel before the purification process was higher than $600 \mathrm{ppm}$. As showing in Fig.10. Typically, the separation of biodiesel and glycerol mixture at volume ratio of 5:3 was completed at $70 \mathrm{~s}$ that means most of high molecular weight of glycerol was disposed to the bottom but suspending light particles was required more time to precipitation. Therefore, it is essentially to purify biodiesel by water-washing.

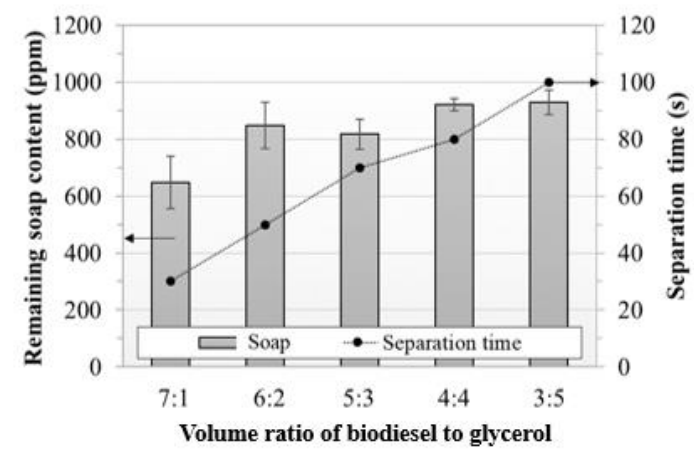

Fig. 10. Separation efficiency and the remaining soap content before the purification process with water-washing with the use of EC with applied AC voltage of $96 \mathrm{~V}$ using Al point-to-point electrodes and the inter-electrode distance of $0.1 \mathrm{~cm}$ varying volume ratios of biodiesel to glycerol.

\subsubsection{Effect of remaining catalyst content}

The remaining base-catalyst in the upper biodiesel-rich phase was completely eliminated after GS for at least $24 \mathrm{hr}$ or overnight. Electrocoagulation is intended to speed up the elimination of remaining catalyst and soap contents. However, it took at least $3000 \mathrm{~s}(50 \mathrm{~min})$ to completely eliminate the remaining catalyst content for the volume ratio of biodiesel to glycerol 2:6.

\subsection{Comparison between gravitational settling and electrocoagulation separation processes}

The performance of EC process was compared to the GS for the separation time, the methyl ester and remaining soap contents.

\subsubsection{Separation time}

The separation of biodiesel and glycerol with the use of EC was faster by $1.4-6.6$ times in comparing with the GS at any volume ratios of biodiesel to glycerol as shown in Fig. 11. The separation time was increasing as increasing the volume of glycerol due to its viscosity. Basically, the electric field attracted glycerol and other polar molecules closely. Then, diffuse layer of electrical charge was compressed by rising ionic strength between two electrodes. After that, those molecules created inter-droplet bridging [21]. Finally, they were settled and formed glycerol layer. But at 2:6 volume ratio of biodiesel to glycerol 
ratio, the separation time was sharply increased because large amount glycerol caused the viscosity to be higher. So, polar molecules were unable to move and coagulate.

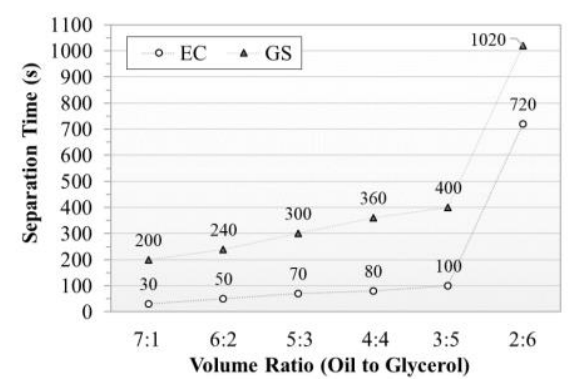

Fig. 11. Performance comparison of separation process between GS and EC with applied AC voltage of $96 \mathrm{~V}$ using Al point-topoint electrodes and the inter-electrode distance of $0.1 \mathrm{~cm}$ varying the volume ratios of biodiesel to glycerol.

\subsubsection{Remaining soap content}

As demonstrated in Fig. 12, the electrocoagulation could significantly reduce the soap content at a greater amount than the gravitational settling by 6 times, but not completely eliminated since there were light-weight glycerol and other particles suspending in the mixture. Even the clear interface of two layers was observed but those particles would take more time to separate. Still, it reduced the separation time by 4 times.

\subsubsection{Methyl ester content}

According to biodiesel standard of EN 14214 and ASTM D6751, the ester content is supposed to be more than $96.5 \%$ and remaining soap content must be less than $5 \mathrm{ppm}$ in order to prevent clogged fuel filter. Hence, biodiesel needs to be washed by DI water to remove soap and unconverted chemicals such as monoglyceride, diglyceride and triglyceride. As Fig. 13 shown that, the electrocoagulation could separate the glycerol from biodiesel and the methyl ester content was 95.91\% before the purification process with water-washing. By washing with DI water, the methyl ester content was improved to $98.56 \%$ after $2^{\text {nd }}$ washing and could remove all unreacted residue. In contrast, with the gravitational settling separation method, it was essential to wash at least 4 times to meet EN 14214 standard and obtain the methyl ester content of $97.72 \%$ and 5 times to obtain the same result as with the electrocoagulation.

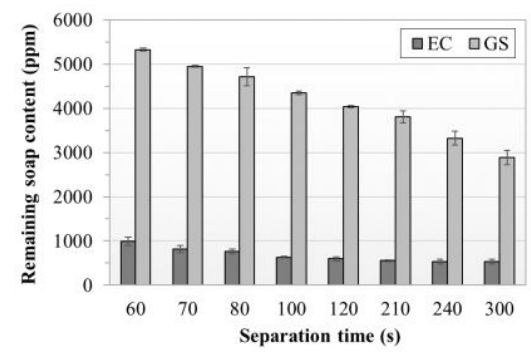

Fig. 12. Comparison of remaining soap content before the purification process with water-washing for two separation processes at the separation efficiency of $100 \%$ : GS and EC with applied $\mathrm{AC}$ voltage of $96 \mathrm{~V}$ using $\mathrm{Al}$ point-to-point electrodes and the inter-electrode distance of $0.1 \mathrm{~cm}$ at the volume ratio of biodiesel to glycerol of 5:3.

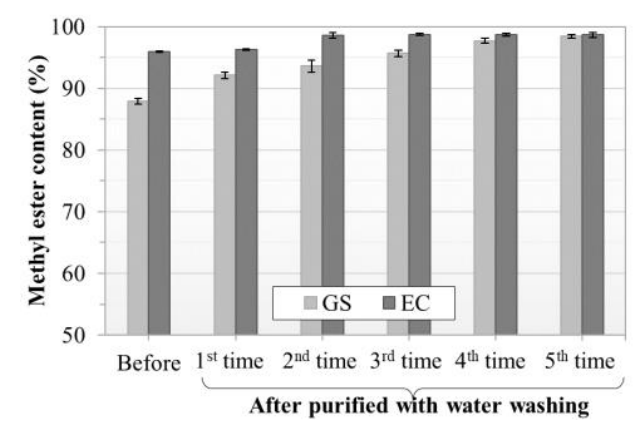

Fig. 13. Comparison of methyl ester content before and after the purification process with water-washing for two separation processes at the separation efficiency of $100 \%$ : GS and EC with applied $\mathrm{AC}$ voltage of $96 \mathrm{~V}$ using $\mathrm{Al}$ point-to-point electrodes and the inter-electrode distance of $0.1 \mathrm{~cm}$ at the volume ratio of biodiesel to glycerol of 5:3.

\section{Conclusion}

It was demonstrated that EC was efficient method to separate glycerol from biodiesel with applied AC at low voltage using $\mathrm{Al}$ point-to-point electrodes at the interelectrode distance of $0.1 \mathrm{~cm}$. The separation time was reduced up to 6 times compared to GS. Efficiency of separation was increased with higher volume ratio of biodiesel to glycerol of 3:5 (62.5\% of glycerol content), however, the efficiency was declined rapidly with higher glycerol content. In addition, most of remaining soap in biodiesel phase was eliminated lower 800 ppm within 70 $\mathrm{s}$ but not the catalyst. To completely eliminate the catalyst, higher electrical stress was required. Besides EC was environment-friendly since it could reduce wastewater from washing step. Methyl ester was purified to $95.91 \%$ by EC without purification. Nevertheless, the methyl ester content was improved significantly to $98.56 \%$ by 2 times of water-washing.

In conclusion, EC with AC low voltage was capable for biodiesel separation from glycerol in comparing with the system of high voltage (data not shown). However, to be commercialized with larger capacity, many of this unit must be implemented to provide sufficient electrical stress for the separation glycerol as well as other residues. This would result in higher energy consumption. Economic analysis is recommended. Moreover, to completely remove the remaining soap and catalyst, the electrical stress must be increased to the range of $\mathrm{kV}$. Therefore, $\mathrm{EC}$ with $\mathrm{AC}$ high voltage may be more practical [18].

The authors would like to thank King Mongkut's University of Technology North Bangkok (Research Grant Contract No. KMUTNB-GOV-58-17) for the financial support.

\section{References}

1 P.L. Castro Verdezoto, J.A. Vidoza, W.L.R. Gallo, Energy Policy, 134, 110-948 (2019)

2 C. Kochaphum, S.H. Gheewala, S. Vinitnantharat, Energy Sustain. Dev. 17, 658-670 (2013)

3 I.M. Atadashi, M.K. Aroua, A.R. Abdul Aziz, N.M.N. 
Sulaiman, Egypt J Pet. 24, 383-396 (2015)

4 J.U. Putra, L. Kalsum, Y. Bow, J Fundam Appl Chem, 3(3), 89-93 (2018)

5 F.L. Lobo, H. Wang, T. Huggins, J. Rosenblum, K.G. Linden, Z.J. Ren, J Hazard Mater 309, 180-184 (2016)

6 A. Abbaszadeh, B. Ghobadian, G. Najafi, Adv Environ Biol. 8, 321-324 (2014)

7 F. Barbieri Gonzaga, S. Pereira Sobral, Talanta. 97, 199203 (2012)

8 BS EN14103:2011, Fat and oil derivatives - Fatty Acid Methyl Esters (FAME)-Determination of ester and linolenic acid methyl ester contents (BSI. London, Gt. Britain, 2011))

9 M.Y. Ong, K.W. Chew, P.L. Show, S. Nomanbhay, Energy Convers Manag. (2019).

10 European Stardard EN14105, Fat and oil derivativesFatty Acid Methyl Esters (FAME)-Determination of free and total glycerol and mo, di-, triglyceride contents Australia Standards Institute: Wein, (2011).

11 P. Wanking, A.F. Muhtadin, T. Kangsadan, (International Conference Proceeding of PACCON, 2019).

12 K. Vuthivat, T. Kangsadan, S. Chalermwisutkul, (International Conference Proceeding of PACCON, 2012).
13 W. Garnwitayee, T. Kangsadan, S. Chalermwisutkul, ICBEE, (2012).

14 P. Cañizares, F. Martínez, C. Jiménez, C. Sáez, M.A Rodrigo, J Hazard Mater. 151, 44-51 (2008).

15 A. Gupta, H.B. Eral, T.A. Hatton, P.S. Doyle, Soft Matter. 12, 2826-2841 (2016).

16 X. Luo, H. Yin, J. Ren, H. Yan, Y. Lü, L. He, J Phys Chem. C. 123, 19588-19595 (2019).

17 A.A. Cerqueira, P.S.A. Souza, M.R.C. Marques, Brazilian J Chem Eng. 31, 693-701 (2014).

19 T. Chungcharoen, K. Netjaibun, T. Pratabkong, P. Suwannasam, W. Limmun, Energy Procedia, 2017.

20 J.M. White, P. Shah, S. Sanford, M. Valverde, G. Meier, Renew Energy (2010).

21 G. Knothe, J Am Oil Chem Soc. 83, 823-833 (2006).

22 S. Banerjee, S. Mazumdar, Int J Anal Chem. 2012, 140 (2012).

23 P. Cañizares, F. Martínez, C. Jiménez, C. Sáez, M. A.Rodrigo, J Hazard Mater. 151(1), 44-51 (2008)

24 A. Abbaszadeh, B. Ghobadian, G. Najafi, Adv Environ Biol. 8, 321-324 (2014).

25 A.A. Cerqueira, P.S.A. Souza, M.R.C. Marques, Brazilian J Chem Eng. 31, 693-701 (2014). 